Sharif University of Technology
Scientia Iranica
SCIENTIA
I RAN I CA

\title{
Reliable seismic collapse assessment of short-period structures using new proxies for ground motion record selection
}

\author{
M. Yakhchalian ${ }^{a, *}$, G. Ghodrati Amiri ${ }^{\mathrm{b}}$ and M. Eghbali \\ a. Department of Civil Engineering, Qazvin Branch, Islamic Azad University, Qazvin, P.O. Box 34185-1416, Iran. \\ b. Center of Excellence for Fundamental Studies in Structural Engineering, School of Civil Engineering. Iran University of Science \\ \& Technology, Tehran, P.O. Box 16765-163, Iran. \\ c. School of Civil Engineering, Iran University of Science \& Technology, P.O. Box 16765-163, Tehran, Iran.
}

Received 19 November 2015; received in revised form 27 May 2016; accepted 9 July 2016

\author{
KEYWORDS \\ Ground motion record \\ selection; \\ Short-period \\ structures; \\ Reliable seismic \\ collapse assessment; \\ Epsilon; \\ Gamma; \\ Fragility curve.
}

\begin{abstract}
One of the important steps in seismic collapse assessment of structures, using nonlinear dynamic analyses, is the appropriate selection of ground motion records. Epsilon $\left(\varepsilon_{S a}\right)$, eta $(\eta)$, and gamma $(\gamma)$ for long-period structures are proxies recently proposed for Ground Motion Record Selection (GMRS). In this study, two parameters, named $\gamma_{s}$, are proposed, which have considerable correlation with the collapse capacity of shortperiod structures having fundamental period less than 1 sec. One of these parameters is a linear combination of $\varepsilon_{S a}$, epsilon of Pseudo Spectral Acceleration (PSA) at 1.5 times of the fundamental period of the structure $\left(\varepsilon_{S a\left(1.5 T_{1}\right)}\right)$, and $\varepsilon_{\mathrm{PGV}}$. The other one is a linear combination of $\varepsilon_{S a}, \varepsilon_{S a\left(1.5 T_{1}\right)}$ and epsilon of spectrum intensity, $\varepsilon_{S I}$. To obtain and optimize $\gamma_{s}$, the Particle Swarm Optimization (PSO) algorithm is applied. Since the parameters proposed as $\gamma_{s}$ have significant correlation with the collapse capacity of short-period structures, they can be used as efficient proxies for GMRS in seismic collapse assessment of short-period structures. The results show that GMRS using $\gamma_{s}$ leads to a reduction in the dispersion of structural collapse capacity in comparison with GMRS based on $\varepsilon_{S a}$ or $\eta$.

(C) 2017 Sharif University of Technology. All rights reserved.
\end{abstract}

\section{Introduction}

Assessing the probability of collapse is an important part of Performance-Based Earthquake Engineering (PBEE). Selection of appropriate ground motion records is a key issue in reliable collapse simulation of structures by using nonlinear dynamic analyses. In fact, the structural collapse under high-amplitude

*. Corresponding author. Tel.: +982833231706 E-mail addresses: m_yakhchalian@alumni.iust.ac.ir ( $M$. Yakhchalian); ghodrati@iust.ac.ir (G. Ghodrati Amiri); m_eghbali@iust.ac.ir (M. Eghbali).

doi: $10.24200 /$ sci. 2017.4162 ground motions is significantly affected by ground motion spectral characteristics. According to Baker and Cornell [1] and Haselton et al. [2], for a given ground motion hazard level, the shape of Uniform Hazard Spectrum (UHS) can considerably differ from the spectral shape of a real ground motion record, which has an equally high spectral amplitude at a single period. In other words, in UHS, the spectral accelerations in all periods have the same exceedance probability, and because the joint occurrence of these spectral accelerations, with the same exceedance probability, is very unlikely, the spectral shape of UHS and that of a real ground motion are considerably different. Therefore, it is important to account for the spectral shape of rare ground motions in the Ground 
Motion Record Selection (GMRS) for reliable seismic collapse assessment of structures.

Baker [3] showed that structural response and collapse capacity are dependent on epsilon $\left(\varepsilon_{S a}\right)$ values of ground motion records, which are used for seismic response analyses. Parameter $\varepsilon_{S a}$ represents the number of standard deviations that the logarithmic spectral acceleration at the fundamental period of the structure, $\ln S a\left(T_{1}\right)$, for a certain ground motion record is away from the average value estimated for the records of the same general characteristics by a Ground Motion Prediction Equation (GMPE) [3,4]; it can be obtained as follows:

$$
\varepsilon_{S a}=\frac{\ln S a\left(T_{1}\right)-\mu_{\ln S a}}{\sigma_{\ln S a}}
$$

where $\ln S a\left(T_{1}\right)$ is the natural logarithm of observed $S a\left(T_{1}\right)$ in a particular ground motion record; $\mu_{\ln S a}$ and $\sigma_{\ln S a}$ are the predicted mean and standard deviation of $\ln S a\left(T_{1}\right)$, respectively. In fact, $\varepsilon_{S a}$ is a spectral shape indicator at the fundamental period of the structure, $T_{1}$. Based on the studies by Baker and Cornell $[1,3,5]$, the effect of $\varepsilon_{S a}$ on structural response is more distinctive than those of magnitude and distance. They found that the mean collapse capacity of a structure increases with increasing $\varepsilon_{S a}$ of ground motions used for collapse simulations, and this increase may be significant. Other researchers also pointed out the key effect of spectral shape in seismic collapse assessment (e.g., [6-9]). Therefore, $\varepsilon_{S a}$ can be used as a proxy for GMRS to account for the effect of spectral shape, corresponding to a target seismic event, in seismic collapse assessment of structures. To use $\varepsilon_{S a}$ as a proxy for GMRS, a set of ground motion records having a mean $\varepsilon_{S a}$ value equal to target $\varepsilon_{S a}$, obtained from seismic hazard disaggregation $[10,11]$, should be selected. After selection of ground motion records with regard to $\varepsilon_{S a}$, $S a\left(T_{1}\right)$ can be used as a scalar Intensity Measure (IM). This GMRS procedure leads to reliable seismic collapse assessment of structures by reduction in the bias and dispersion of structural collapse capacity.

Increasing the reliability of seismic collapse assessments by using an efficient GMRS procedure motivates researchers to propose more efficient proxies for GMRS. To propose an efficient proxy for GMRS, Mousavi et al. [12] investigated the linear combinations of $\varepsilon_{S a}$ with epsilons of different peak ground IMs (i.e., PGA, PGV, and PGD) to be used instead of $\varepsilon_{S a}$. They proposed a new proxy termed "eta" $(\eta)$, which is a linear combination of $\varepsilon_{S a}$ and the peak ground velocity epsilon, $\varepsilon_{\mathrm{PGV}}$ (see Eq. (2)). They showed that $\eta$ has higher correlation with the collapse capacity of structures, compared with $\varepsilon_{S a}$, and its use in GMRS instead of $\varepsilon_{S a}$ leads to a considerable reduction in the dispersion of structural collapse capacity prediction. It should be noted that the amount of this reduction is related to the size of the correlation between the proxy parameter (i.e., $\eta$ ) and the structural collapse capacity:

$$
\eta=\varepsilon_{S a}-0.823 \varepsilon_{\mathrm{PGV}}
$$

Yakhchalian et al. [13] also used the idea of combining epsilons of different IMs to propose an efficient proxy for GMRS in seismic collapse assessment of tall buildings. In addition to $\varepsilon_{S a}$ and epsilons of peak ground IMs, they used Displacement Spectrum Intensity (DSI) [14] epsilon, $\varepsilon_{\mathrm{DSI}}$. They investigated different linear combinations of epsilons to find a combination having higher correlation with the collapse capacity of long-period structures, when compared with $\varepsilon_{S a}$ and $\eta$, and proposed a new proxy termed "gamma" $(\gamma)$ which is a linear combination of $\varepsilon_{S a}$ and $\varepsilon_{\text {DSI }}$ for GMRS in seismic collapse assessment of long-period structures (see Eq. (3)). When a structure subjected to a ground motion is close to collapse, its period increases considerably and its collapse capacity is dependent on the spectral characteristics of the ground motion record in the period range of its near-collapse period. Due to the fact that DSI represents the severity of longperiod ground motion amplitudes, parameter $\gamma$ has high correlation with the structural collapse capacity:

$$
\gamma=\varepsilon_{S a}-0.861 \varepsilon_{\mathrm{DSI}} .
$$

Typically, there are two general methods for reliable seismic collapse assessment of structures by using nonlinear dynamic analyses. The first method, as described above, is accounting for the spectral shape in GMRS procedure and using a common Intensity Measure (IM) for the seismic analyses. The second one is using advanced IMs (e.g., [9,15-18]), which are able to account for the effect of spectral shape in seismic analyses. The present study focuses on the first method to obtain efficient proxies for GMRS in seismic collapse assessment of short-period structures by combining epsilons of different IMs. After investigating different combinations of epsilons, two new parameters, named $\gamma_{s}$, were proposed for structures having fundamental period less than 1 sec. These parameters have better correlation with the collapse capacity of short-period structures when compared with $\varepsilon_{S a}$ and $\eta$. The purpose of this study is to complete the previous research publication by Yakhchalian et al. [13] on the subject of GMRS for reliable seismic collapse assessment of structures.

\section{Calculating epsilons of IMs}

To obtain the optimal combinations of epsilons that have considerable correlation with the collapse capacity of short-period structures, epsilons of IMs should be calculated. The considered IMs are pseudo spectral 
accelerations at the fundamental period of the structure and two larger periods (i.e., $S a\left(T_{1}\right), S a\left(1.5 T_{1}\right)$, and $S a\left(2 T_{1}\right)$ ), peak ground IMs (i.e., PGA, PGV, and PGD), and integral-based IMs such as Acceleration Spectrum Intensity (ASI), Spectrum Intensity (SI), and Displacement Spectrum Intensity (DSI). The integralbased IMs are defined as $[14,19,20]$ :

$$
\begin{aligned}
& \mathrm{ASI}=\int_{0.1}^{0.5} S a(T) d T, \\
& \mathrm{SI}=\int_{0.1}^{2.5} S v(T) d T, \\
& \mathrm{DSI}=\int_{2}^{5} S d(T) d T,
\end{aligned}
$$

where $S a(T), S v(T)$, and $S d(T)$ are the $5 \%$ damped pseudo-acceleration, pseudo-velocity, and displacement response spectra, respectively. ASI, SI, and DSI are indicators of the severity of short-, moderate-, and long-period content of ground motions, respectively.

Calculation of epsilon is common for spectral acceleration $\left(\varepsilon_{S a}\right)$, but epsilons of different IMs, which have GMPEs, can also be calculated. In fact, the epsilon of an IM $\left(\varepsilon_{\mathrm{IM}}\right)$ is the normalized residual for the IM and can be calculated as:

$$
\varepsilon_{\mathrm{IM}}=\frac{\ln \mathrm{IM}-\mu_{\mathrm{ln} \mathrm{IM}}}{\sigma_{\ln \mathrm{IM}}},
$$

where $\ln$ IM is the natural logarithm of the IM observed in a particular ground motion; $\mu_{\ln I M}$ and $\sigma_{\text {ln IM }}$ are the predicted mean and standard deviation of ln IM, which are functions of the earthquake rupture and site of interest. In this study, the Campbell and Bozorgnia GMPE [21] that has been developed as part of the Next Generation Attenuation of Ground Motions (NGA) project for the prediction of $S a(T)$, PGA, PGV, and PGD was used for the calculation of $\varepsilon_{S a}, \varepsilon_{S a\left(1.5 T_{1}\right)}, \varepsilon_{S a\left(2 T_{1}\right)}, \varepsilon_{\mathrm{PGA}}, \varepsilon_{\mathrm{PGV}}$, and $\varepsilon_{\mathrm{PGD}}$ values. To calculate $\varepsilon_{\mathrm{IM}}$ values for the integral-based IMs (i.e., $\varepsilon_{\mathrm{ASI}}, \varepsilon_{\mathrm{SI}}$, and $\varepsilon_{\mathrm{DSI}}$ ), their GMPEs are required. Therefore, the method proposed by Bradley [14,19] and Bradley et al. [20] for the prediction of ASI, SI, and DSI using the GMPE for $S a(T)$ was used.

\section{Collapse capacity of SDOF systems}

To investigate the efficiency of different combinations of $\varepsilon_{\text {IM }}$ parameters as a proxy for GMRS, a set containing 54 nonlinear Single-Degree-Of-Freedom (SDOF) systems created in OpenSees [22] was used. Nine period values of $T=0.2,0.3,0.4,0.5,0.6,0.7,0.8,0.9$ and $1.0 \mathrm{sec}$ were considered for the SDOF systems, and six ductility values of $\mu=2,4,6,8,10$, and 12 were as- sumed for each period. The Bilinear model [23] was applied to model the SDOF systems, and cyclic deterioration was neglected. The post-yield hardening stiffness ratio was considered equal to 0.02 , and the ductility to the onset of negative stiffness was assumed to be 0.9 of the total ductility of the SDOF system $\left(\mu_{c}=0.9 \mu\right)$. Moreover, a mass proportional damping ratio of 0.05 was considered for all of the SDOF systems.

Nowadays, Incremental Dynamic Analysis (IDA) [24] is a favorable method for seismic performance assessment of structures, used by many researchers. To obtain collapse capacities of the SDOF systems, IDAs were performed by using a set containing 67 ground motion records, applied by Yakhchalian et al. [13]. The selected records were taken from the PEER NGA database [25]. In this study, a procedure was used to overcome the convergence problems that occur in the nonlinear dynamic analyses. This procedure gradually decreases the time step size for analysis, $d t$, until the solution converges. If decreasing $d t$ does not lead to convergence, then the procedure uses other solution algorithms in the OpenSees. If changing the solution algorithm and also decreasing $d t$ for each solution algorithm still does not lead to convergence, then the procedure increases the test tolerance to converge the solution. To perform IDA, the amplitude of each record, $S a\left(T_{1}\right)$, was scaled to an increasing intensity until it causes collapse. The collapse occurs when an SDOF system reaches its zero strength and final ductility [26]. Consequently, the collapse capacity, $S a_{\text {col }}$, was obtained for each ground motion record. The Hunt and Fill algorithm [24] was used to obtain the collapse capacity values corresponding to each of the SDOF systems. This algorithm is a tracing algorithm that increases $S a\left(T_{1}\right)$ by a constant step until the collapse is reached, and then uses smaller steps to increase the accuracy of the IDA curve. Having the collapse capacity values for the SDOF systems, the correlation between different combinations of $\varepsilon_{\text {IM }}$ parameters and the collapse capacity can be investigated.

\section{Obtaining efficient proxies for GMRS}

In this study, knowing that each $\varepsilon_{\mathrm{IM}}$ can represent part of information hidden in a given ground motion record, different combinations of $\varepsilon_{\text {IM }}$ parameters were investigated to propose an efficient predictor of structural collapse capacity. In fact, to have an efficient proxy for GMRS in seismic collapse assessment of short-period structures, the proposed proxy parameter should be an efficient predictor of structural collapse capacity $\left(S a_{\text {col }}\right)$, i.e. appropriately correlated with $\ln S a_{\text {col }}$. The size of correlation between a proxy parameter and $\ln S a_{\text {col }}$ shows the ability of the proxy parameter to reduce the dispersion of collapse capacity prediction (record-to-record variability) when used for GMRS. 
Yakhchalian et al. [13] showed that $\varepsilon_{S a}$ is more efficient than other $\varepsilon_{\mathrm{IM}}$ parameters, considered alone, to predict the collapse capacity of SDOF systems having period greater than $1 \mathrm{sec}$. Similarly, this issue can be examined in the case of short-period SDOF systems. Figure 1 shows the correlation between the collapse capacity of an SDOF system and parameters $\varepsilon_{S a}$ and $\varepsilon_{\mathrm{ASI}}$. It can be seen that $\ln S a_{\mathrm{col}}$ is more correlated with $\varepsilon_{S a}$ than $\varepsilon_{\mathrm{ASI}}$. Such a result was also observed for $\varepsilon_{\mathrm{IM}}$ parameters other than $\varepsilon_{\mathrm{ASI}}$. To propose efficient proxies being highly correlated with the structural collapse capacity, different linear combinations of $\varepsilon_{\mathrm{IM}}$ parameters were investigated as $\gamma_{s}$. Eq. (8) indicates the general form considered for $\gamma_{s}$ :

$$
\begin{aligned}
\gamma_{s}= & \varepsilon_{S a}+k_{1} \varepsilon_{S a\left(1.5 T_{1}\right)}+k_{2} \varepsilon_{S a\left(2 T_{1}\right)}+k_{3} \varepsilon_{\mathrm{PGA}} \\
& +k_{4} \varepsilon_{\mathrm{PGV}}+k_{5} \varepsilon_{\mathrm{PGD}}+k_{6} \varepsilon_{\mathrm{ASI}}+k_{7} \varepsilon_{\mathrm{SI}}+k_{8} \varepsilon_{\mathrm{DSI}} .
\end{aligned}
$$

It should be mentioned that all of $\varepsilon_{\mathrm{IM}}$ parameters, shown in Eq. (8), are not necessary to exist in a combination. For each of the assumed combinations, values of coefficients $k_{i}$ were optimized by Particle Swarm Optimization (PSO) algorithm [27] to make the combination appropriately correlated with the collapse capacity of the considered SDOF system. In a combination of $\varepsilon_{\mathrm{IM}}$ parameters, the optimum coefficients determined for each SDOF system are dependent on the structural characteristics such as period and ductility. For each SDOF system, assuming a lognormal distribution for the collapse capacity values obtained from IDAs, the values of $\ln S a_{\text {col }}$ were normalized to the standard form with a zero mean and unit variance. Then, to eliminate the dependency of the optimized $\gamma_{s}$ on the structural characteristics, similar to the method used by Yakhchalian et al. [13], the optimization process was performed on the whole values of normalized $\ln S a_{\mathrm{col}}$ for all of the SDOF systems.

The Kolmogorov-Smirnov (KS) test [28] was applied to check the assumption of lognormal distribution for $S a_{\text {col }}$ values corresponding to each of the SDOF systems. In general, the KS test is used to investigate if the distribution of a random variable (here, $S a_{\mathrm{col}}$ ) can be approximated using a specific distribution (e.g., lognormal distribution), and its result is a $p$-value. Typically, a $p$-value greater than 0.05 , obtained from the KS test, confirms the assumed distribution for $S a_{\mathrm{col}}$ values. The results of the KS tests, for all of the SDOF systems, confirmed the lognormal distribution of $S a_{\text {col }}$. As an instance, Figure 2 presents the IDA curves, collapse capacity points, normalized backbone curve, and the Probability Density Function (PDF) of a lognormal distribution fitted on the normalized histogram of $S a_{\mathrm{col}}$ for an SDOF system. It can be seen that there is a good resemblance between the normalized histogram of $S a_{\mathrm{col}}$ and the PDF of lognormal distribution; the $p$-value obtained from the KS test is greater than 0.05 .

After normalizing $\ln S a_{\mathrm{col}}$ values for all of the SDOF systems to the standard form, a vector of size $3618(54 \times 67)$ for the normalized $\ln S a_{\text {col }}$ values was obtained. In addition, according to Section 2, nine vectors with the same size for $\varepsilon_{S a}, \varepsilon_{S a\left(1.5 T_{1}\right)}, \varepsilon_{S a\left(2 T_{1}\right)}$, $\varepsilon_{\mathrm{PGA}}, \varepsilon_{\mathrm{PGV}}, \varepsilon_{\mathrm{PGD}}, \varepsilon_{\mathrm{ASI}}, \varepsilon_{\mathrm{SI}}$, and $\varepsilon_{\mathrm{DSI}}$ corresponding to the vector of collapse capacity values were obtained. To achieve the maximum correlation between the vector of normalized $\ln S a_{\text {col }}$ values and each combination of $\varepsilon_{\text {IM }}$ parameters, the PSO algorithm was applied. Table 1 presents the optimum coefficients obtained for different combinations and the corresponding Pearson correlation [29] coefficients $(\rho)$. It can be seen that $\varepsilon_{S a}$, as a structural collapse capacity predictor, has the highest correlation with the collapse capacity of the SDOF systems, compared with the other single $\varepsilon_{\mathrm{IM}}$ parameters. However, adding other $\varepsilon_{\mathrm{IM}}$ parameters to $\varepsilon_{S a}$, in a combination, can result in a more efficient predictor of structural collapse capacity. The results show that combination 11 , containing $\varepsilon_{S a}$ and $\varepsilon_{\mathrm{PGV}}$, and combination 14 , containing $\varepsilon_{S a}$ and $\varepsilon_{\text {SI }}$, are more efficient than combination 15 , which contains $\varepsilon_{S a}$ and $\varepsilon_{\mathrm{DSI}}$. The reason for this issue is

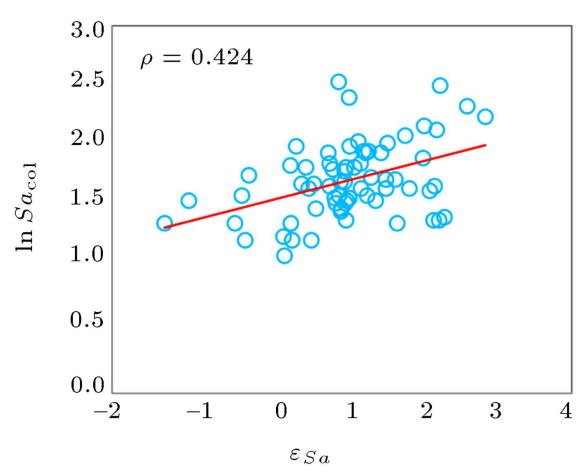

(a)

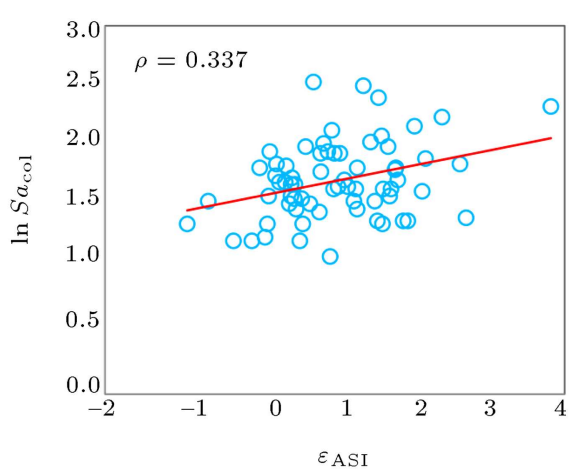

(b)

Figure 1. Correlation between the collapse capacity of an SDOF system $(T=0.5 \mathrm{sec}$ and $\mu=4)$ and epsilons of intensity measures: (a) $\varepsilon_{S a}$ and (b) $\varepsilon_{\text {ASI. }}$. 


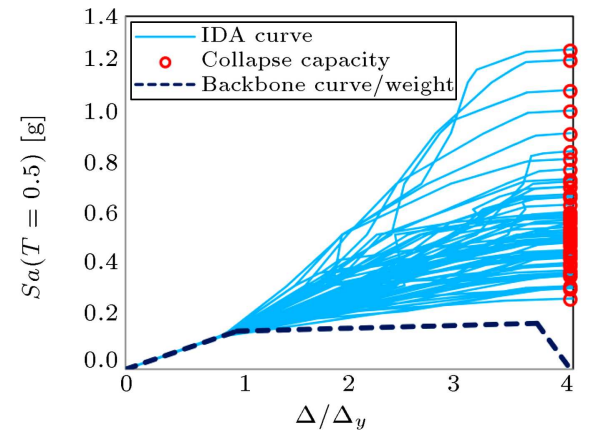

(a)

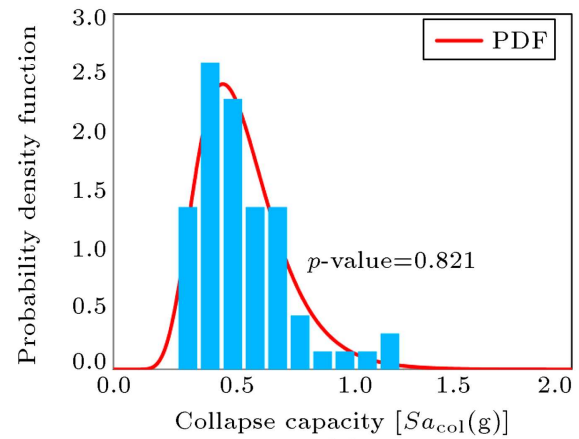

(b)

Figure 2. (a) IDA curves, collapse capacity points and normalized backbone curve. (b) PDF of a lognormal distribution fitted on the normalized histogram of $S a_{\mathrm{col}}$, for an SDOF system with $T=0.5 \mathrm{sec}, \mu=4$ and the yield strength to weight ratio $F_{y} / W=0.15$.

Table 1. Results of optimization for different combinations of $\varepsilon_{\mathrm{IM}}$ parameters and the corresponding correlation coefficients.

\begin{tabular}{|c|c|c|c|c|c|c|c|c|c|c|}
\hline No. & $\varepsilon_{S a}$ & $\varepsilon_{S a\left(1.5 T_{1}\right)}$ & $\varepsilon_{S a\left(2 T_{1}\right)}$ & $\varepsilon_{\mathrm{PGA}}$ & $\varepsilon_{\mathrm{PGV}}$ & $\varepsilon_{\mathrm{PGD}}$ & $\varepsilon_{\mathrm{ASI}}$ & $\varepsilon_{\mathrm{SI}}$ & $\varepsilon_{\mathrm{DSI}}$ & $\rho$ \\
\hline 1 & 1 & - & - & - & - & - & - & - & - & 0.443 \\
\hline 2 & - & 1 & - & - & - & - & - & - & - & 0.005 \\
\hline 3 & - & - & 1 & - & - & - & - & - & - & 0.133 \\
\hline 4 & - & - & - & 1 & - & - & - & - & - & 0.159 \\
\hline 5 & - & - & - & - & 1 & - & - & - & - & 0.102 \\
\hline 6 & - & - & - & - & - & 1 & - & - & - & 0.119 \\
\hline 7 & - & - & - & - & - & - & 1 & - & - & 0.236 \\
\hline 8 & - & - & - & - & - & - & - & 1 & - & 0.066 \\
\hline 9 & - & - & - & - & - & - & - & - & 1 & 0.217 \\
\hline 10 & 1 & - & - & -0.427 & - & - & - & - & - & 0.489 \\
\hline 11 & 1 & - & - & - & -0.836 & - & - & - & - & 0.639 \\
\hline 12 & 1 & - & - & - & - & -0.591 & - & - & - & 0.506 \\
\hline 13 & 1 & - & - & - & - & - & -0.221 & - & - & 0.452 \\
\hline 14 & 1 & - & - & - & - & - & - & -0.789 & - & 0.652 \\
\hline 15 & 1 & - & - & - & - & - & - & - & -0.721 & 0.570 \\
\hline 16 & 1 & -0.718 & - & - & - & -- & - & - & - & 0.632 \\
\hline 17 & 1 & - & -0.735 & - & - & - & - & - & - & 0.657 \\
\hline 18 & 1 & -0.359 & -0.437 & & & & & & & 0.679 \\
\hline 19 & 1 & -0.685 & - & - & - & - & -0.059 & - & - & 0.633 \\
\hline 20 & 1 & -0.385 & - & - & - & - & - & -0.47 & - & 0.691 \\
\hline 21 & 1 & -0.604 & - & - & - & - & - & - & -0.318 & 0.680 \\
\hline 22 & 1 & - & -0.701 & - & - & - & -0.06 & - & - & 0.658 \\
\hline 23 & 1 & - & -0.397 & - & - & - & - & -0.436 & - & 0.686 \\
\hline 24 & 1 & - & -0.615 & - & - & - & - & - & -0.269 & 0.680 \\
\hline 25 & 1 & - & - & -0.328 & - & - & - & - & -0.531 & 0.597 \\
\hline 26 & 1 & - & - & - & -0.767 & - & - & - & -0.093 & 0.640 \\
\hline 27 & 1 & - & - & - & - & -0.014 & - & - & -0.712 & 0.570 \\
\hline 28 & 1 & - & - & -0.073 & - & - & - & -0.734 & - & 0.653 \\
\hline 29 & 1 & - & - & - & -0.382 & - & - & -0.488 & - & 0.671 \\
\hline 30 & 1 & - & - & - & - & 0.006 & - & -0.792 & - & 0.652 \\
\hline 31 & 1 & - & - & - & -1.009 & 0.263 & - & - & - & 0.651 \\
\hline 32 & 1 & -0.612 & - & -0.171 & - & - & - & - & - & 0.644 \\
\hline 33 & 1 & -0.449 & - & - & -0.474 & - & - & - & - & 0.704 \\
\hline 34 & 1 & -0.658 & - & - & - & -0.2 & - & - & - & 0.647 \\
\hline 35 & 1 & - & -0.629 & -0.166 & - & - & - & - & - & 0.665 \\
\hline 36 & 1 & - & -0.451 & - & -0.461 & - & - & - & - & 0.704 \\
\hline 37 & 1 & - & -0.685 & - & - & -0.144 & - & - & - & 0.662 \\
\hline 38 & 1 & -0.275 & -0.231 & -0.082 & -0.44 & 0.207 & 0.087 & -0.04 & -0.1 & 0.732 \\
\hline
\end{tabular}


that PGV and SI indicate the severity of moderateperiod content of ground motion records, whereas DSI indicates the severity of long-period content of ground motion records. When a short-period structure is close to collapse, its elongated period is in the range of moderate periods; thus, $\varepsilon_{\mathrm{PGV}}$ and $\varepsilon_{\mathrm{SI}}$ have more effect on its collapse capacity compared with $\varepsilon_{\mathrm{DSI}}$. When combining at least two $\varepsilon_{\mathrm{IM}}$ parameters, the obtained coefficient for each $\varepsilon_{\mathrm{IM}}$ expresses the weight of that $\varepsilon_{\mathrm{IM}}$ in the considered combination. For example, in combination 30 , which contains $\varepsilon_{S a}, \varepsilon_{\mathrm{SI}}$, and $\varepsilon_{\mathrm{PGD}}$, the obtained coefficient for $\varepsilon_{\mathrm{PGD}}$ (i.e., 0.006) indicates that $\varepsilon_{\mathrm{PGD}}$ has negligible effect on the achieved correlation coefficient, when compared with $\varepsilon_{S a}$ and $\varepsilon_{\text {SI }}$. The results indicate that combination 20 (containing $\varepsilon_{S a}$, $\varepsilon_{S a\left(1.5 T_{1}\right)}$, and $\varepsilon_{\mathrm{SI}}$ ), combination 33 (containing $\varepsilon_{S a}$, $\varepsilon_{S a\left(1.5 T_{1}\right)}$, and $\varepsilon_{\mathrm{PGV}}$ ), and combination 36 (containing $\varepsilon_{S a}, \varepsilon_{S a\left(2 T_{1}\right)}$, and $\left.\varepsilon_{\mathrm{PGV}}\right)$ have comparatively high correlation with the structural collapse capacity. It should be noted that combinations 33 and 36 result in the same correlation coefficients, and also the obtained coefficients for $\varepsilon_{S a\left(1.5 T_{1}\right)}$ and $\varepsilon_{S a\left(2 T_{1}\right)}$ in these combinations are approximately the same. It can therefore be inferred that using $\varepsilon_{S a\left(1.5 T_{1}\right)}$ or $\varepsilon_{S a\left(2 T_{1}\right)}$ does not lead to significant difference in the results. Thus, from these two combinations, combination 33 was selected. Furthermore, combination 38, which contains all of considered $\varepsilon_{\text {IM }}$ parameters, has the highest correlation coefficient. However, its correlation coefficient is not

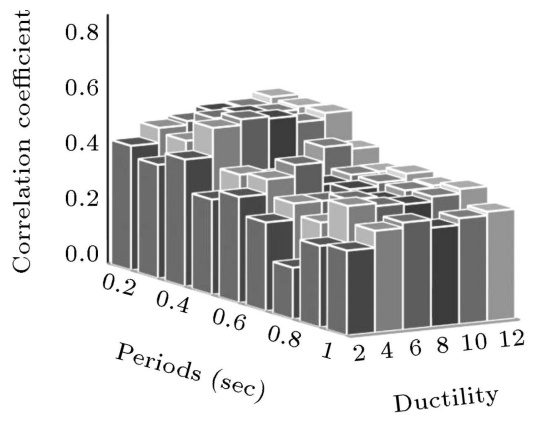

(a)

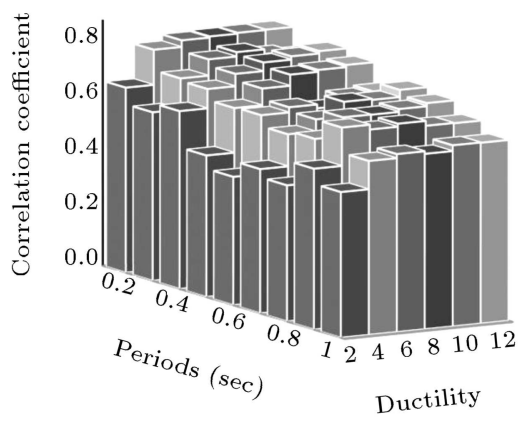

(c) significantly higher than those from combinations 20 and 33, which are simpler. Therefore, combinations 20 and 33 , due to their high correlation and simplicity (using fewer $\varepsilon_{\text {IM }}$ parameters), were selected as the two efficient predictors of structural collapse capacity. The proposed parameters as $\gamma_{s}$ are presented in Eq. (9):

$$
\gamma_{s}=\varepsilon_{S a}-0.449 \varepsilon_{S a\left(1.5 T_{1}\right)}-0.474 \varepsilon_{\mathrm{PGV}}
$$

or:

$$
\gamma_{s}=\varepsilon_{S a}-0.385 \varepsilon_{S a\left(1.5 T_{1}\right)}-0.47 \varepsilon_{\mathrm{SI}} .
$$

Figure 3(a)-(d) illustrate the correlation coefficients between $\ln S a_{\text {col }}$ values of the SDOF systems and $\varepsilon_{S a}$, $\eta$ and $\gamma_{s}$, respectively. It can be seen that the proposed parameters as $\gamma_{s}$ are more efficient than $\varepsilon_{S a}$ and $\eta$ for predicting the structural collapse capacity.

Mousavi et al. [12] used the genetic algorithm [30] to propose parameter $\eta$. As described previously, in this study, the PSO algorithm was used to optimize coefficients $k_{i}$ in each combination of $\varepsilon_{\mathrm{IM}}$ parameters. In combination 11 , which contains $\varepsilon_{S a}$ and $\varepsilon_{\mathrm{PGV}}$, the value obtained for the coefficient of $\varepsilon_{\mathrm{PGV}}$ is -0.836 . This value is very similar to that of -0.826 obtained by Mousavi et al. [12] as the coefficient of $\varepsilon_{\mathrm{PGV}}$ in parameter $\eta$. Thus, considering the differences between the SDOF systems and ground motion records used in both studies, it can be inferred that the optimization algorithm used does not have significant effect on the results.

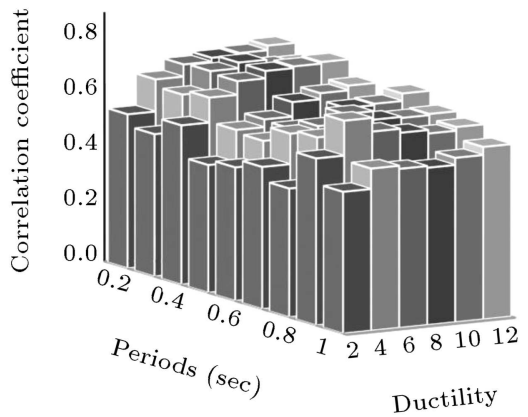

(b)

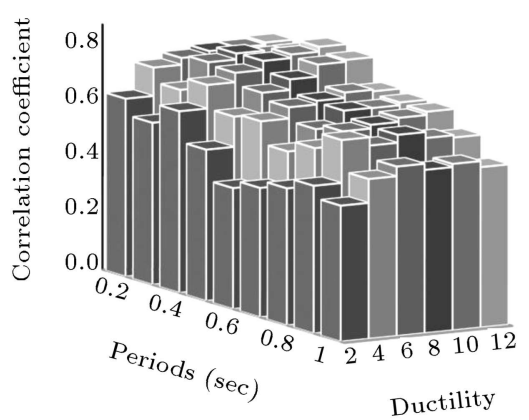

(d)

Figure 3. Correlation coefficients between the collapse capacity of the SDOF systems: (a) $\varepsilon_{S a}$, (b) $\eta$, (c) $\gamma_{s}=\varepsilon_{S a}$ $-0.449 \varepsilon_{S a\left(1.5 T_{1}\right)}-0.474 \varepsilon_{\mathrm{PGV}}$, and (d) $\gamma_{s}=\varepsilon_{S a}-0.385 \varepsilon_{S a\left(1.5 T_{1}\right)}-0.47 \varepsilon_{\mathrm{SI}}$. 


\section{Using $\gamma_{s}$ for collapse capacity prediction of an MDOF structure}

To investigate the efficiency of $\gamma_{s}$ for predicting the collapse capacity of short-period structures, a MultiDegree-Of-Freedom (MDOF) 6-story structure with a fundamental period of $0.6 \mathrm{sec}$ was considered. It is one of the two-dimensional generic one-bay frames designed by Medina and Krawinkler [31] and also used by Ibarra and Krawinkler [32]. The structural model was created in OpenSees. The nonlinear behavior was modeled by using rotational springs at both beam ends and the bottom end of the first-story columns. Bilinear model [23] was used to specify the nonlinear momentrotation behavior of the rotational springs, and cyclic deterioration was neglected. For each member, the post-yield and negative post-capping stiffness ratios of 0.03 and -0.1 were assumed, respectively, and the member ductility capacity value, $\delta_{c} / \delta_{y}$, was considered to be 4 . For nonlinear time history analyses, $5 \%$ Rayleigh damping was assigned to the first mode and the mode at which the cumulative mass participation exceeds $95 \%$. Furthermore, the $P-\Delta$ effect was considered in modeling.

The IDA approach was applied to determine the collapse capacity of the structure. The far-field ground motion record set, used for analyzing the SDOF systems, was used to perform IDAs. The collapse was assumed to occur when the IDA curve becomes flat, i.e. the maximum inter-story drift ratio of the structure reaches 0.15 . Figure 4 illustrates the IDA curves of the 6-story structure. Figure 5 shows the comparison between the efficiency of $\gamma_{s}, \varepsilon_{S a}$, and $\eta$ for predicting the collapse capacity of the considered structure. It can be seen that using both parameters proposed as $\gamma_{s}$ for collapse capacity prediction of the structure results in higher efficiency (correlation coefficients of 0.77 and 0.8 ) in comparison to using $\varepsilon_{S a}$ and $\eta$, resulting in correlation coefficients of 0.51 and 0.67 , respectively. It can therefore be concluded that both $\gamma_{s}$ parameters

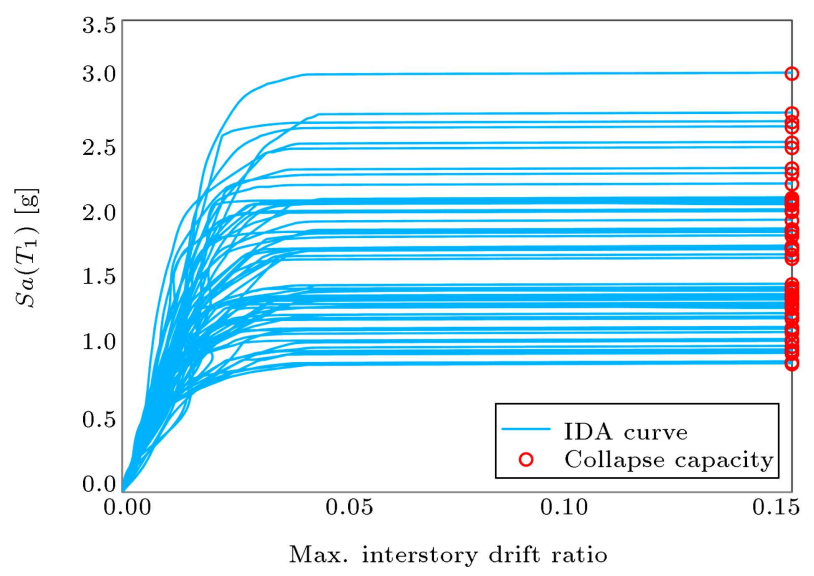

Figure 4. IDA curves of the 6-story structure. are more efficient than $\varepsilon_{S a}$ and $\eta$ for collapse capacity prediction of short-period structures.

\section{Target value for $\gamma_{s}$}

In order to assess seismic collapse of structures, in a specific seismic hazard level, using both $\gamma_{s}$ parameters as proxies for GMRS, target values of $\varepsilon_{S a\left(1.5 T_{1}\right)}, \varepsilon_{\mathrm{PGV}}$, and $\varepsilon_{\text {SI }}$ are required. When the target value of $\varepsilon_{\text {Sa }}$, determined from seismic hazard disaggregation, is known, the target value of $\varepsilon_{S a\left(1.5 T_{1}\right)}$ can be obtained as:

$$
\varepsilon_{S a\left(1.5 T_{1}\right)}^{*}=\varepsilon_{S a}^{*} \times \rho_{\varepsilon_{S a}, \varepsilon_{S a\left(1.5 T_{1}\right)}},
$$

where $\varepsilon_{S a\left(1.5 T_{1}\right)}^{*}$ and $\varepsilon_{S a}^{*}$ are the target values of $\varepsilon_{S a\left(1.5 T_{1}\right)}$ and $\varepsilon_{S a}$, respectively; $\rho_{\varepsilon_{S a}, \varepsilon_{S a\left(1.5 T_{1}\right)}}$ is the correlation between $\varepsilon_{S a}$ and $\varepsilon_{S a\left(1.5 T_{1}\right)}$, which can be obtained using the relationship proposed by Baker and Jayaram [33] for calculating the correlation of spectral accelerations. To determine target $\varepsilon_{\mathrm{PGV}}$, the empirical equation proposed by Mousavi et al. [12] can be used. Moreover, similar to the study performed by Yakhchalian et al. [13], the target value of $\varepsilon_{\mathrm{SI}}$ can be determined based on a relationship between $\varepsilon_{S a}$ and $\varepsilon_{\text {SI }}$. To obtain such a relationship, a set containing 350 horizontal ground motion records related to shallow crustal earthquakes, used by Yakhchalian et al. [13] to determine the empirical relationship between $\varepsilon_{S a}$ and $\varepsilon_{\mathrm{DSI}}$, was considered.

Figure 6 illustrates the correlation between $\varepsilon_{S a}$ and $\varepsilon_{\mathrm{SI}}$ in two periods of 0.4 and $0.8 \mathrm{sec}$, obtained using the set containing 350 ground motion records. It can be seen that the estimated regression coefficients and correlation coefficient vary by period. Figure 7 shows the variations of correlation coefficient between $\varepsilon_{S a}$ and $\varepsilon_{\text {SI }}$ by period. It can be seen that by increasing the period, the correlation between the two epsilons increases. Assuming that there is a linear relationship between $\varepsilon_{\mathrm{SI}}$ and $\varepsilon_{S a}$ as presented in Eq. (11), the coefficients of this equation, $a(T)$ and $b(T)$, can be determined as the functions of period:

$$
\varepsilon_{\mathrm{SI}}=a(T) \varepsilon_{S a}+b(T) .
$$

In this study, second- and third-order regressions were used to determine $a(T)$ and $b(T)$ as functions of period, respectively. Figure 8 shows these regression analyses that led to the following equations for $a(T)$ and $b(T)$ :

$$
\begin{aligned}
& a(T)=-0.494 T^{2}+1.029 T+0.308 \\
& b(T)=-0.483 T^{3}+1.05 T^{2}-0.729 T+0.205 .
\end{aligned}
$$

Therefore, considering target $\varepsilon_{S a}$, the target values of $\varepsilon_{S a\left(1.5 T_{1}\right)}, \varepsilon_{\mathrm{SI}}, \varepsilon_{\mathrm{PGV}}$, and thus $\gamma_{s}$ can be calculated. Then, the target values of both $\gamma_{s}$ parameters can be used for GMRS. 


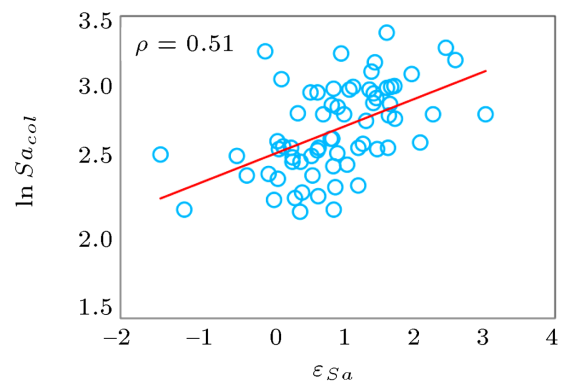

(a)

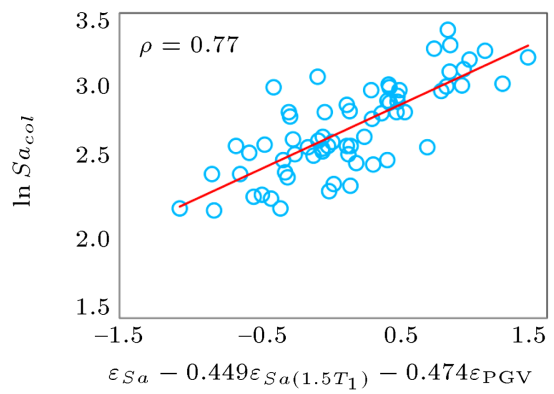

(c)

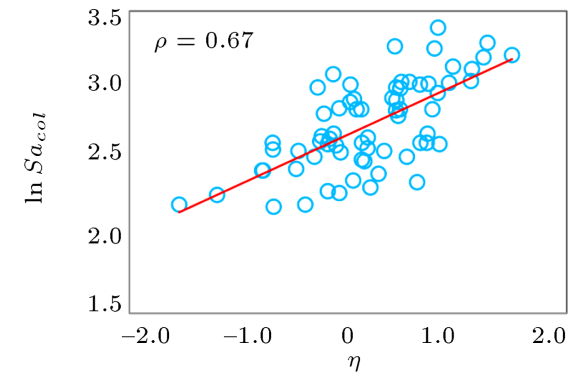

(b)

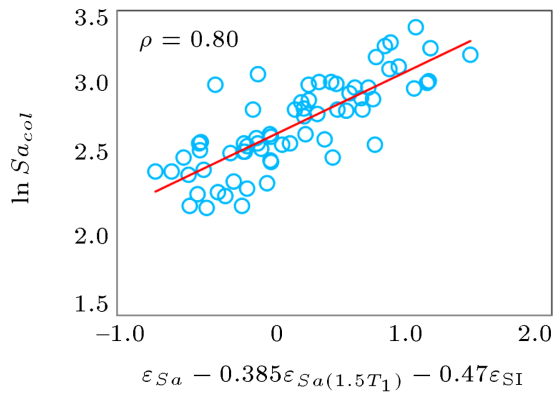

(d)

Figure 5. Comparison between the efficiency of different parameters for collapse capacity prediction of the 6-story structure: (a) $\varepsilon_{S a}$, (b) $\eta$, (c) $\gamma_{s}=\varepsilon_{S a}-0.449 \varepsilon_{S a\left(1.5 T_{1}\right)}-0.474 \varepsilon_{\mathrm{PGV}}$, and (d) $\gamma_{s}=\varepsilon_{S a}-0.385 \varepsilon_{S a\left(1.5 T_{1}\right)}-0.47 \varepsilon_{\mathrm{SI}}$.

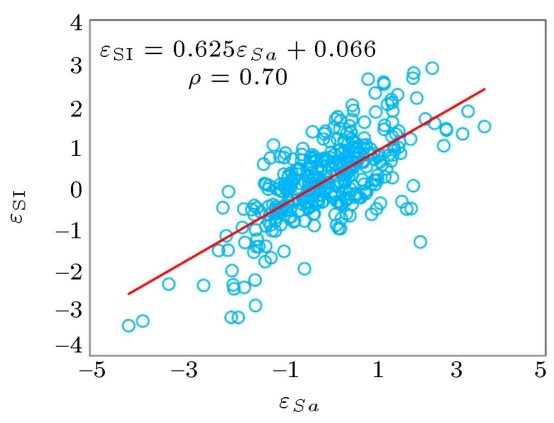

(a)

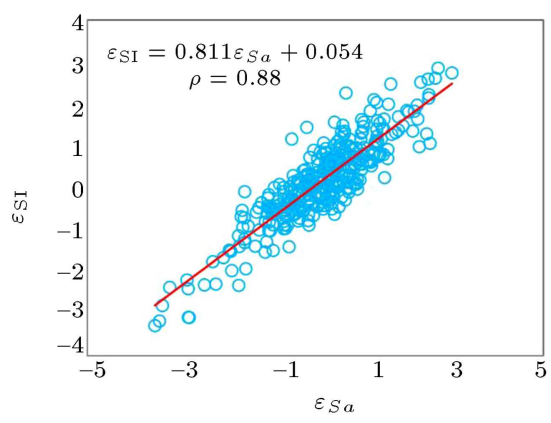

(b)

Figure 6. Correlation between $\varepsilon_{S a}$ and $\varepsilon_{\mathrm{SI}}$ at periods of (a) $0.4 \mathrm{sec}$ and (b) $0.8 \mathrm{sec}$.

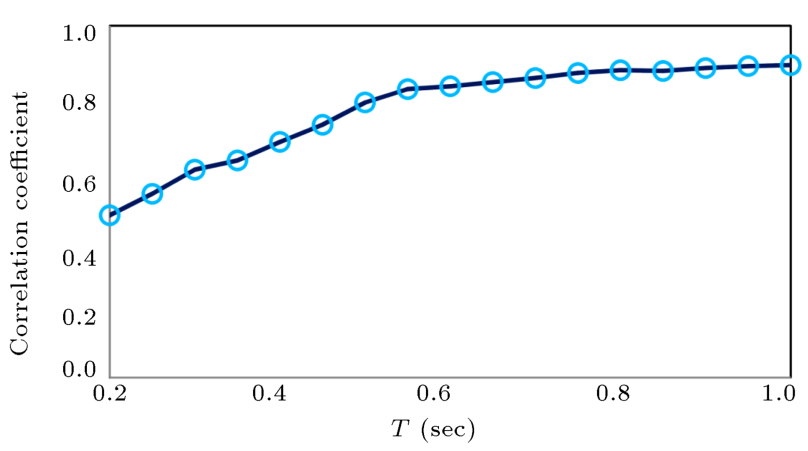

Figure 7. Correlation coefficients between $\varepsilon_{S a}$ and $\varepsilon_{\mathrm{SI}}$ at different periods.

\section{GMRS and seismic collapse assessment}

In this section, different GMRS procedures (based on $\gamma_{s}, \eta$, and $\left.\varepsilon_{S a}\right)$ are investigated for seismic collapse assessment of the 6-story structure. For this purpose, two seismic hazard levels with target $\varepsilon_{S a}$ values of 0.8 and 1.7 were considered. As mentioned previously, target values of $\gamma_{s}$ and $\eta$ can be obtained based on target $\varepsilon_{S a}$. It should be mentioned that the normalized form of $\eta$, proposed by Mousavi et al. [12], was used. Therefore, the target value of $\eta$ was equal to the target value of $\varepsilon_{S a}$. In addition, by using the procedure described in Section 6, the target values for the first formulation of $\gamma_{s}$ corresponding to target $\varepsilon_{S a}$ values of 0.8 and 1.7 were obtained as equal to 0.102 and 0.33 , respectively. The target values for the second formulation of $\gamma_{s}$ corresponding to the considered target $\varepsilon_{S a}$ values were also obtained as equal to 0.236 and 0.527 , respectively.

The target values of $\gamma_{s}, \eta$, and $\varepsilon_{S a}$ were used as proxies for GMRS, and four bins (two bins for $\gamma_{s}$ ) 


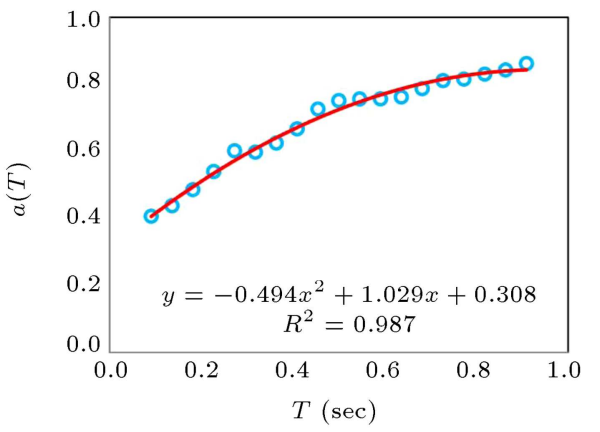

(a)

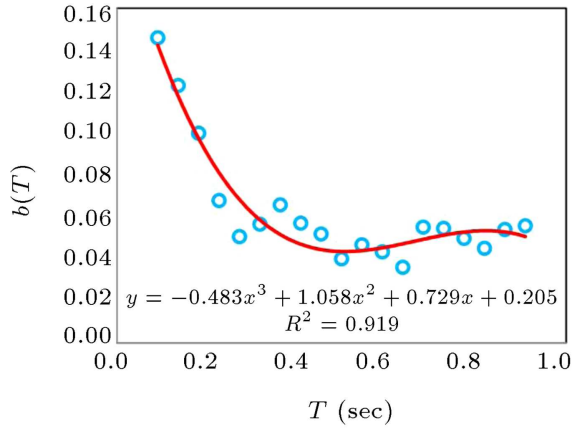

(b)

Figure 8. Obtaining $a(T)$ and $b(T)$ as functions of period using (a) second- and (b) third-order regressions.

containing 20 ground motion records were selected for each of the seismic hazard levels. The ground motion records in each bin were selected to satisfy the criterion that the mean value of the corresponding proxy parameter should be equal to the target value determined for the assumed seismic hazard level. Additionally, the following criteria were also considered in GMRS:

- Magnitude $\geq 6.5$;

- The closest distance to rupture between 10 and $100 \mathrm{~km}$;

- Soil $V_{s 30}$ (average shear wave velocity in top $30 \mathrm{~m}$ of the site profile) between 180 and $750 \mathrm{~m} / \mathrm{s}$.

After GMRS, IDAs were performed using the selected ground motion records in each bin to obtain the collapse capacity of the structure. The KS test [28] was applied to verify the lognormal distribution of the obtained collapse capacity values for each bin. The results of the KS tests confirmed the lognormality assumption for collapse capacity values. Considering this assumption, collapse fragility curves were obtained for each GMRS procedure. Figure 9 shows the collapse fragility curves obtained by using the proxy parameters for GMRS considering the two seismic hazard levels.
In addition, these fragility curves are compared with the fragility curve obtained using the general far-field ground motion record set containing 67 records. It can be seen that using the proxies for GMRS leads to different fragility curves, implying that the obtained logarithmic means and standard deviations used for plotting the fragility curves are different. Table 2 presents the median of $S a_{\text {col }}$, which is the exponential of the mean of $\ln S a_{\mathrm{col}}$ values, and its corresponding logarithmic standard deviation, $\sigma_{\ln S a_{\mathrm{col}}}$, for each of the GMRS procedures. It can be seen that using the proxy parameters for GMRS leads to reduction in $\sigma_{\ln S a_{\text {col }}}$ values, compared with the use of the general far-field ground motion record set. Furthermore, using each of the proposed $\gamma_{s}$ parameters as a proxy for GMRS results in a lower $\sigma_{\ln S a_{\text {col }}}$ than those obtained from using $\eta$ and $\varepsilon_{S a}$ for both of the target values. It should be mentioned that the logarithmic standard deviation of collapse capacity values, $\sigma_{\ln S a_{\text {col }}}$, determines the slope of the collapse fragility curve. A lower value of $\sigma_{\ln S a_{\text {col }}}$ represents a steeper collapse fragility curve. Thus, when using an efficient proxy parameter that results in a lower value of $\sigma_{\ln S a_{\mathrm{col}}}$, the obtained collapse fragility curve is steeper and more reliable than those obtained by using less efficient proxies parameters.

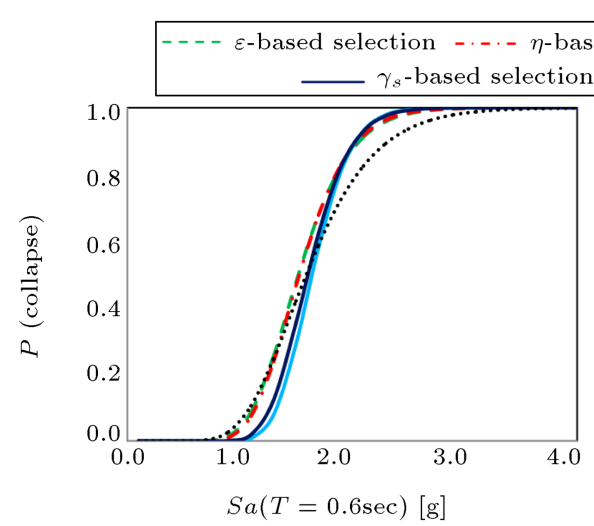

(a)

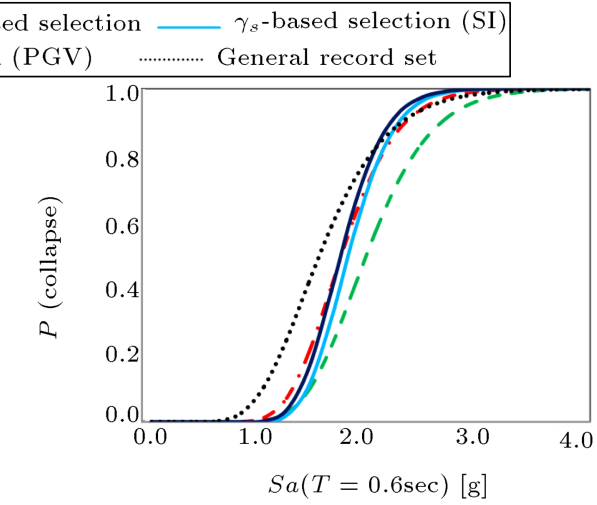

(b)

Figure 9. Collapse fragility curves obtained for the 6-story structure using the ground motion record bins selected based on the considered proxies: (a) Target $\varepsilon_{S a}$ value $=0.8$ and (b) target $\varepsilon_{S a}$ value $=1.7$. 
Table 2. Results of the collapse assessment of the 6-story structure considering different proxies $\left(\varepsilon_{S a}, \eta\right.$ and $\left.\gamma_{s}\right)$ for GMRS.

\begin{tabular}{|c|c|c|c|c|c|c|c|c|c|}
\hline \multirow{2}{*}{$\begin{array}{c}\text { Target } \varepsilon_{S a} \\
\text { value }\end{array}$} & \multicolumn{4}{|c|}{0.8} & \multicolumn{4}{|c|}{1.7} & \multirow{2}{*}{$\begin{array}{l}\text { General } \\
\text { record set }\end{array}$} \\
\hline & $\varepsilon_{S a}$ & $\eta$ & $\gamma_{s}(\mathrm{SI})$ & $\gamma_{s}(\mathrm{PGV})$ & $\varepsilon_{S a}$ & $\eta$ & $\gamma_{s}(\mathrm{SI})$ & $\gamma_{s}(\mathrm{PGV})$ & \\
\hline Median of $S a_{\mathrm{col}}[\mathrm{g}]$ & 1.522 & 1.530 & 1.644 & 1.606 & 2.010 & 1.788 & 1.849 & 1.781 & 1.592 \\
\hline$\sigma_{\ln S} S a_{\mathrm{col}}$ & 0.238 & 0.228 & 0.165 & 0.182 & 0.224 & 0.217 & 0.170 & 0.175 & 0. 296 \\
\hline
\end{tabular}

In order to continue this study, epsilons of other intensity measures can be used to propose optimized combinations of $\varepsilon_{\mathrm{IM}}$ parameters as efficient proxies for ground motion record selection in seismic collapse assessment of different types of structural systems.

\section{Conclusions}

In this study, two parameters were proposed as $\gamma_{s}$ to be used as proxies for GMRS in seismic collapse assessment of short-period structures. Each of these proxies is a linear combination of different $\varepsilon_{\mathrm{IM}}$ parameters. One of them is a combination of $\varepsilon_{S a}, \varepsilon_{S a\left(1.5 T_{1}\right)}$, and $\varepsilon_{\mathrm{SI}}$; the other one is a combination of $\varepsilon_{S a}, \varepsilon_{S a\left(1.5 T_{1}\right)}$, and $\varepsilon_{\mathrm{PGV}}$. The PSO algorithm was used in proposing $\gamma_{s}$ parameters. It was shown that for the short-period SDOF and MDOF structures having fundamental period less than $1 \mathrm{sec}$, each of these parameters has higher correlation with the structural collapse capacity, when compared with $\varepsilon_{S a}$ and $\eta$. Because of their efficiency for structural collapse capacity prediction, $\gamma_{s}$ parameters were used as proxies for GMRS. To determine the target value for $\gamma_{s}$ parameter including $\varepsilon_{\mathrm{SI}}$, an empirical equation was proposed to obtain $\varepsilon_{\mathrm{SI}}$ for a given $\varepsilon_{S a}$. To investigate the efficiency of $\gamma_{s}$ parameters as proxies for GMRS in seismic collapse assessment of short-period structures, a 6-story moment-resisting frame with a fundamental period of $0.6 \mathrm{~s}$ was used. It was shown that using each of the proposed proxies for GMRS leads to a reduction in the structural collapse capacity dispersion, when compared with using $\varepsilon_{S a}$ and $\eta$, and therefore a more reliable collapse fragility curve can be obtained.

\section{References}

1. Baker, J.W. and Cornell, C.A. "A vector-valued ground motion intensity measure consisting of spectral acceleration and epsilon", Earthq. Eng. and Struct. D., 34(10), pp. 1193-1217 (2005).

2. Haselton, C.B., Baker, J.W., Liel, A.B. and Deierlein, G.G. "Accounting for ground motion spectral shape characteristics in structural collapse assessment through an adjustment for epsilon", J. of Struct. Eng., 137(3), pp. 332-344 (2011).
3. Baker, J.W. "Vector-valued ground motion intensity measures for probabilistic seismic demand analysis", Ph.D. dissertation, Department of Civil and Environmental Engineering, Stanford University, Stanford, CA (2005).

4. Kazantzi, A.K. and Vamvatsikos, D. "Intensity measure selection for vulnerability studies of building classes", Earthq. Eng. and Struct. D., 44(15), pp. 2677-2694 (2015).

5. Baker, J.W. and Cornell, C.A. "Spectral shape, epsilon and record selection", Earthq. Eng. and Struct. D., 35(9), pp. 1077-1095 (2006).

6. Haselton, C.B. and Baker, J.W. "Ground motion intensity measures for collapse capacity prediction: Choice of optimal spectral period and effect of spectral shape", Proc. of 8th National Conf. on Earthq. Eng., Oakland, CA (2006).

7. Zareian, F. "Simplified performance based earthquake engineering", Ph.D. dissertation, Department of Civil and Environmental Engineering, Stanford University, Stanford, CA (2006).

8. Goulet, C.A., Haselton, C.B., Mitrani-Reiser, J., Beck, J.L., Deierlein, G.G., Porter, K.A. and Stewart, J.P. "Evaluation of the seismic performance of a codeconforming reinforced-concrete frame building-From seismic hazard to collapse safety and economic losses", Earthq. Eng. and Struct. D., 36(13), pp. 1973-1997 (2007).

9. Eads, L., Miranda, E. and Lignos, D. "Spectral shape metrics and structural collapse potential", Earthq. Eng. and Struct. D., 45(10), pp. 1643-1659 (2016).

10. McGuire, R.K. "Probabilistic seismic hazard analysis and design earthquakes: closing the loop", B. of the Seismol. Soc. of Am., 85(5), pp. 1275-1284 (1995).

11. Bazzurro, P. and Cornell, C.A. "On disaggregation of seismic hazard", B. of the Seismol. Soc. of Am., 89(2), pp. 501-520 (1999).

12. Mousavi, M., Ghafory-Ashtiany, M. and Azarbakht, A. "A new indicator of elastic spectral shape for the reliable selection of ground motion records", Earthq. Eng. and Struct. D., 40(12), pp. 1403-1416 (2011).

13. Yakhchalian, M., Ghodrati Amiri, G. and Nicknam, A. "A new proxy for ground motion selection in seismic collapse assessment of tall buildings", The Struct. Des. of Tall and Spec., 23(17), pp. 1275-1293 (2014). 
14. Bradley, B.A. "Empirical equations for the prediction of displacement spectrum intensity and its correlation with other intensity measures", Soil Dyn. and Earthq. Eng., 31(8), pp. 1182-1191 (2011).

15. Marafi, N.A., Berman, J.W. and Eberhard, M.O. "Ductility-dependent intensity measure that accounts for ground-motion spectral shape and duration", Earthq. Eng. and Struct. D., 45(4), pp. 653-672 (2016).

16. Yakhchalian, M., Nicknam, A. and Ghodrati Amiri, G. "Proposing an optimal integral-based intensity measure for seismic collapse capacity assessment of structures under pulse-like near-fault ground motions", J. of Vibroeng., 16(3), pp. 1360-1375 (2014).

17. Yakhchalian, M., Nicknam, A. and Ghodrati Amiri, G. "Optimal vector-valued intensity measure for seismic collapse assessment of structures", Earthq. Eng. and Eng. Vib., 14(1), pp. 37-54 (2015).

18. Haj Najafi, L. and Tehranizadeh, M. "New intensity measure parameter based on record's velocity characteristics", Sci. Iran., 22(5), pp. 1674-1691 (2015).

19. Bradley, B.A. "Site-specific and spatially distributed ground-motion prediction of acceleration spectrum intensity", B. of the Seismol. Soc. of Am., 100(2), pp. 792-801 (2010).

20. Bradley, B.A., Cubrinovski, M., MacRae, G.A. and Dhakal, R.P. "Ground-motion prediction equation for SI based on spectral acceleration equations", B. of the Seismol. Soc. of Am., 99(1), pp. 277-285 (2009).

21. Campbell, K.W. and Bozorgnia, Y. "CampbellBozorgnia NGA ground motion relations for the geometric mean horizontal component of peak and spectral ground motion parameters", PEER Report 2007/02, Pacific Earthquake Engineering Research Center, University of California, Berkeley, CA (2007).

22. OpenSees, Open System for Earthquake Engineering Simulation, Pacific Earthquake Engineering Research Center, University of California, Berkeley, CA http://opensees.berkeley.edu (2012).

23. Ibarra, L.F., Medina, R.A. and Krawinkler, H. "Hysteretic models that incorporate strength and stiffness deterioration", Earthq. Eng. and Struct. D., 34(12), pp. 1489-1511 (2005).

24. Vamvatsikos, D. and Cornell, C.A. "Incremental dynamic analysis", Earthq. Eng. and Struct. D., 31(3), pp. 491-514 (2002).

25. Pacific Earthquake Engineering Research Center (PEER), PEER Next Generation Attenuation (NGA) database. http://peer.berkeley.edu/nga. (2008).

26. Ibarra, L. and Krawinkler, H. "Variance of collapse capacity of SDOF systems under earthquake excitations", Earthq. Eng. and Struct. D., 40(12), pp. 12991314 (2011).
27. Poli, R., Kennedy, J. and Blackwell, T. "Particle swarm optimization", Swarm Intell., 1(1), pp. 33-57 (2007).

28. Hogg, R.V. and Ledolter, J., Engineering Statistics, MacMillan, New York (1987).

29. Ang, A.H.S. and Tang, W.H., Probability Concepts in Engineering: Emphasis on Applications in Civil and Environmental Engineering, Wiley: New York (2007).

30. Goldberg, D.E., Genetic Algorithms in Search, Optimization, and Machine Learning, 412, Reading Menlo Park: Addison-Wesley (1989).

31. Medina, R.A. and Krawinkler, H. "Seismic demands for nondeteriorating frame structures and their dependence on ground motions", PEER Report 2003/15, Pacific Earthquake Engineering Research Center, University of California, Berkeley, CA (2003).

32. Ibarra, L.F. and Krawinkler, H. "Global collapse of frame structures under seismic excitations", Report No. 152, The John A. Blume Earthquake Engineering Center, Department of Civil and Environmental Engineering, Stanford University, Stanford, CA (2005).

33. Baker, J.W. and Jayaram, N. "Correlation of spectral acceleration values from NGA ground motion models", Earthq. Spectra, 24(1), pp. 299-317 (2008).

\section{Biographies}

Masood Yakhchalian received his MSc degree in 2010 from Amirkabir University of Technology (AUT) and $\mathrm{PhD}$ degree in Structural Engineering in 2015 from Iran University of Science and Technology (IUST). His main research areas are performance-based earthquake engineering, seismic hazard analysis, and using optimization algorithms in structural engineering.

Gholamreza Ghodrati Amiri received his BSc degree in 1989, MSc degree in 1992 from Sharif University of Technology in Iran, and PhD degree in 1997 from McGill University in Canada. Currently, he is a Professor at Iran University of Science and Technology (IUST). His research studies are about seismic hazard analysis, performance-based earthquake engineering, and structural health monitoring.

Mahdi Eghbali is a $\mathrm{PhD}$ candidate of Structural Engineering in Iran University of Science and Technology (IUST). His current research interests include earthquake engineering, modern nonlinear dynamic analyses, development of nonlinear static analysis methods, seismic probabilistic risk assessment, and modern lateral load resisting systems. 